\title{
2.7 ̊̊ cryo-EM structure of ex vivo RML prion fibrils
}

Szymon W. Manka ${ }^{1}$, Wenjuan Zhang ${ }^{1}$, Adam Wenborn ${ }^{1}$, Jemma Betts ${ }^{1}$, Susan Joiner ${ }^{1}$, Helen R. Saibil ${ }^{2 *}$, John Collinge ${ }^{1 *}$ and Jonathan D.F. Wadsworth ${ }^{1 *}$

${ }^{1} \mathrm{MRC}$ Prion Unit at UCL, Institute of Prion Diseases, University College London, 33 Cleveland Street, London W1W 7FF, UK

${ }^{2}$ Institute of Structural and Molecular Biology, Department of Biological Sciences, Birkbeck College, University of London, Malet Street, London WC1E 7HX, UK

*correspondence to: Helen Saibil (h.saibil@mail.cryst.bbk.ac.uk), John Collinge (jc@prion.ucl.ac.uk) or Jonathan Wadsworth (j.wadsworth@prion.ucl.ac.uk)

\begin{abstract}
Mammalian prions are lethal infectious agents that propagate as distinct strains and are composed of multichain assemblies of misfolded host-encoded prion protein (PrP), often referred to as prion rods. The structural features that define infectious prion rods and the molecular determinants of prion strain diversity are poorly understood. Here, we present a near-atomic resolution cryo-EM structure of PrP fibrils present in highly infectious prion rod preparations isolated from the brains of RML prion-infected mice. We found that prion rods comprise single-protofilament helical amyloid fibrils that coexist with twisted pairs of the same protofilaments. Each rung of the protofilament is formed by a single PrP monomer with the ordered core comprising PrP residues 94-225, which folds to create two asymmetric lobes with the $\mathrm{N}$-linked glycans and the glycosylphosphatidylinositol anchor projecting from the C-terminal lobe. The overall architecture is comparable to that of recently reported PrP fibrils isolated from the brain of hamsters infected with the 263K prion strain. However, there are marked conformational variations that could result from differences in PrP primary sequence and/or represent distinguishing features of the distinct prion strains. These conformational changes impact the overall geometry of the fibrils and may also impact fibril pairing, one or both of which may critically influence PrP glycoform selection that occurs during strain-specific prion propagation.
\end{abstract}

Prions are lethal infectious agents that cause fatal neurodegenerative diseases in mammals, including scrapie in sheep and goats, chronic wasting disease in cervids, bovine spongiform encephalopathy (BSE) in cattle and Creutzfeldt-Jakob disease (CJD) in humans ${ }^{1,2}$. They are devoid of nucleic acid and composed principally or entirely of multichain fibrillar assemblies of misfolded, host-encoded prion protein (PrP), a glycosylphosphatidylinositol (GPI)anchored cell surface glycoprotein containing two asparagine (N)-linked glycosylation sites ${ }^{1-}$ ${ }^{3}$. Prions propagate by means of seeded protein polymerization, which involves recruitment of PrP monomers to fibrillar assemblies followed by fragmentation of these elongating structures to generate more seeds. Different prion strains produce distinct clinicopathological phenotypes in the same inbred host and appear to be encoded by distinct misfolded PrP conformations and assembly states ${ }^{1-3}$. Since the discovery of prions, considerable international effort has been focused on determining their structure in order to understand unique facets of prion biology, including the mechanisms of replication, the 
differences between prions and non-infectious amyloid, the molecular basis of strain diversity, inter-species transmission barriers and toxicity ${ }^{1-4}$. Significantly, the generation of self-propagating polymeric or amyloid protein assemblies is now widely recognised to be involved in the pathogenesis of many other human diseases. Consequently "prion-like" mechanisms of propagation and spread and whether the strain phenomenon is involved in phenotype have become a major research focus in the commoner neurodegenerative diseases $^{3,5-13}$ and recent advances have defined the structures of diverse self-propagating assemblies of tau ${ }^{14-18}$, amyloid- $\beta^{19,20}$ and $\alpha$-synuclein ${ }^{21}$ from human brain.

The structural transition accompanying PrP monomer incorporation into infectious, protease-resistant, detergent-insoluble fibrillar prion assemblies (classically designated as $\operatorname{PrPSc} 1,22,23)$ involves gross rearrangement of the protein fold ${ }^{24}$. While the cellular isoform of $\operatorname{PrP}(\operatorname{PrPC})$ contains an ordered globular C-terminal domain containing three $\alpha$-helices ${ }^{25,26}$, PrP monomers within the infectious prion multimers adopt a $\beta$-strand-rich configuration ${ }^{1,26-}$ ${ }^{29}$ which confers protease-resistance to the C-terminal two-thirds of the protein sequence. The arrangement of $\beta$-strands, potential inclusion of other structural constituents, and the overall architecture of ex vivo prion fibrils (also referred to as prion rods ${ }^{1,4,30}$ ) have been intensely debated on the basis of various indirect, computational or low-resolution structural studies and diverse structural models have been proposed (reviewed in refs ${ }^{4,31}$ ). Notably prion strains indicate structural heterogeneity and may constitute a cloud of diverse molecular assemblies (analogous to a viral quasispecies) 2,32 which further complicates definition of the unifying structural features of a prion. In particular, because strain-specific prion assemblies contain distinct and characteristic ratios of di-, mono- and nonglycosylated $\operatorname{PrP}^{33-37}$, prion architectures must satisfactorily explain how such high-fidelity selection of PrP glycoforms is achieved ${ }^{2,3,38}$.

Cryogenic electron microscopy (cryo-EM) and the recent advances in image processing (Relion) $)^{39-41}$ have enabled direct, high-resolution structural studies of amyloids, fibrillar polymers defined by cross- $\beta$ structure, in which misfolded protein monomers stack to form a ribbon of intermolecular $\beta$-sheets ${ }^{26,42-45}$. Disease-related PrP has long been known to present the tinctorial hallmarks of amyloid ${ }^{30}$ and consistent with this, recent high-resolution cryo-EM studies suggest that protein cores of mammalian prions may generally adopt parallel in-register intermolecular $\beta$-sheet (PIRIBS) amyloid structures ${ }^{46-50}$. In vitro-generated fibrils from recombinant, bacterially derived, full-length human $\mathrm{PrP}^{47}$, an N-terminal fragment thereof ${ }^{48}$, or full-length human E196K $\operatorname{PrP}^{50}$ form such amyloids, each consisting of two symmetrical protofilaments. However, PrP monomers show distinct folds and distinct lateral contacts (inter-protofilament interfaces) in each of these amyloids, which indicates the structural plasticity of PrP, and thus, its potential for adopting different folds in different prion strains, but artificial in vitro polymerisation products have uncertain if any biological relevance. A central problem is that the recombinant PrP substrate is devoid of $\mathrm{N}$-linked glycans and GPI-anchor which may profoundly impact the conformation of the amyloid core and its resultant biological properties. Notably, PrP amyloids formed from recombinant PrP alone are either devoid of detectable prion infectivity or have specific-infectivities far too low for meaningful structural analysis-4,51. Efforts to elucidate prion structure have therefore concentrated on structural characterisation of ex vivo purified material having high specific infectivity, and recently, a cryo-EM study of the hamster $263 \mathrm{~K}$ prion strain 
determined a high-resolution PIRIBS structure of PrP fibrils present in a purified, ex vivo prion sample of high specific infectivity ${ }^{49}$.

In this study we present a $2.7 \AA$ cryo-EM structure of PrP fibrils present in preparations of infectious RML mouse-adapted scrapie prions ${ }^{52,53}$, the most commonly studied laboratory prion strain, purified to extremely high specific infectivity ${ }^{54}$. In vitreous ice we found that infectious prion rods are predominantly single-protofilament helical amyloid fibrils. The protofilaments have a unique PIRIBS conformation, with similarities to fibrils from the $263 \mathrm{~K}$ hamster prion strain, but very different from the PIRIBS conformations of in vitro generated PrP amyloids. Notably, in distinction to purified preparations of the $263 \mathrm{~K}$ hamster prions that were reported to consist of only single protofilaments ${ }^{49}$, in our purified RML prion samples we observed that the single protofilaments coexist with twisted pairs of the same protofilaments. These findings are consistent with our previous low resolution imaging of paired fibres in preparations of extremely high specific infectivity in which we were able to correlate structural entities with infectivity by bioassay of EM grids ${ }^{38,55}$. The presence of both single and paired protofilaments is intriguing and understanding their origins may be critical to elucidating the mechanism of prion propagation and selection of PrP glycoform ratios that distinguish some prion strains.

\section{Results}

Purified RML prion rods show both single and paired protofilament architectures. RML prion rods from the brain of terminally-infected CD1 mice were purified using previously reported methods ${ }^{54}$ with the exception that proteinase $K(P K)$ rather than pronase $E$ was used for initial digestion of brain homogenate. Purified fractions contained disease-related PrP at $\sim 99 \%$ purity with respect to total protein, with all major SDS-PAGE silver-stained bands immuno-reactive with an anti-PrP monoclonal antibody on western blots, which showed the signature PK-resistant fragment size and PrP glycoform ratio that characterises the RML prion strain ${ }^{54}$ (Extended Data Fig. 1). Mass spectrometry analyses of the purified rods showed that PK N-terminally truncates PrP monomers in the rods at residue 88 with no evidence for C-terminal truncation. PK-digested rods thereby comprise PrP monomers starting at residue 89 extending to the C-terminus with intact GPI anchor. Details of these analyses will be published elsewhere. Prion infectivity of purified samples was measured using the Scrapie Cell Assay ${ }^{56}$ as reported previously ${ }^{51,54}$. The specific prion infectivity of all purified samples used in this study corresponds to $\sim 10^{9}$ mouse intra-cerebral $\mathrm{LD}_{50} / \mathrm{mg}$ protein, consistent with our previous findings ${ }^{38,54,55}$. With the exception of very occasional collagen fibres, prion rods were the only visible protein structures in these samples.

The cryo-EM images of frozen-hydrated purified RML prion assemblies revealed two distinct fibrillar morphologies, 10 nm-wide single protofilaments and $20 \mathrm{~nm}$-wide pairs of the same protofilaments. The ratio of observed single protofilaments to pairs was 9:1 (Fig. 1a). The paired protofilaments show clear helical symmetry, with the crossover or half-pitch $\left(180^{\circ}\right.$ helical turn) distance ranging from 150 to $180 \mathrm{~nm}$ (Fig. 1a). The single protofilaments were rarely sufficiently long to encompass the full crossover distance, but this was determined from the reconstruction to be on average slightly shorter (135 $\mathrm{nm}$ ) than the pairs (Fig. 1a and b). 
bioRxiv preprint doi: https://doi.org/10.1101/2021.12.13.472424; this version posted December 14, 2021. The copyright holder for this preprint (which was not certified by peer review) is the author/funder, who has granted bioRxiv a license to display the preprint in perpetuity. It is made available under aCC-BY-NC 4.0 International license.

The presence of predominantly single protofilaments in our infectious RML prion rod preparations was surprising. Based on our previous negative-stain EM and AFM imaging of the purified $\mathrm{RML}$ samples ${ }^{38,55}$, we interpreted the rod preparations to contain predominantly paired fibres. However due to their helical twist the paired fibres, when viewed on a surface, alternate between wider, face-on ( $20 \mathrm{~nm})$ and narrower, edge-on ( $10 \mathrm{~nm}$ ) views. Guided by negative-stain $\mathrm{EM}^{38,55}$ we considered that all narrower views (10 $\mathrm{nm}$ ) seen in 2D projections corresponded to edge-on views of the pairs. With the insight provided by the high-resolution 3D cryo-EM, it is now apparent that the negative-stain 2D EM reflects a distribution of single and double-protofilament architectures (Extended Data Fig. 2).
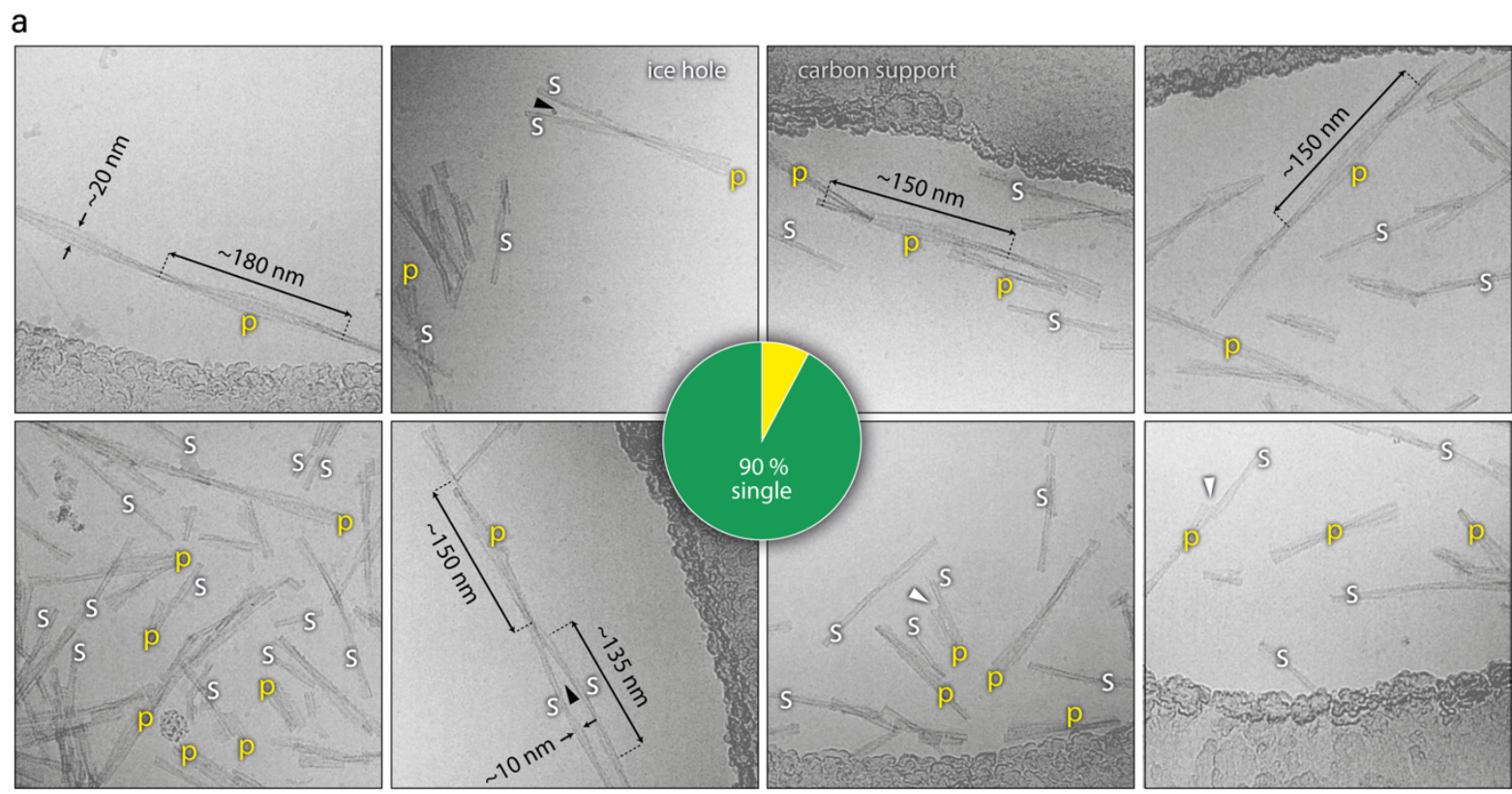

b

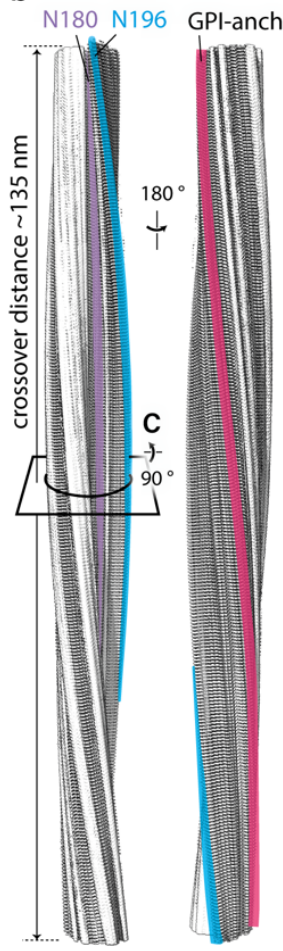

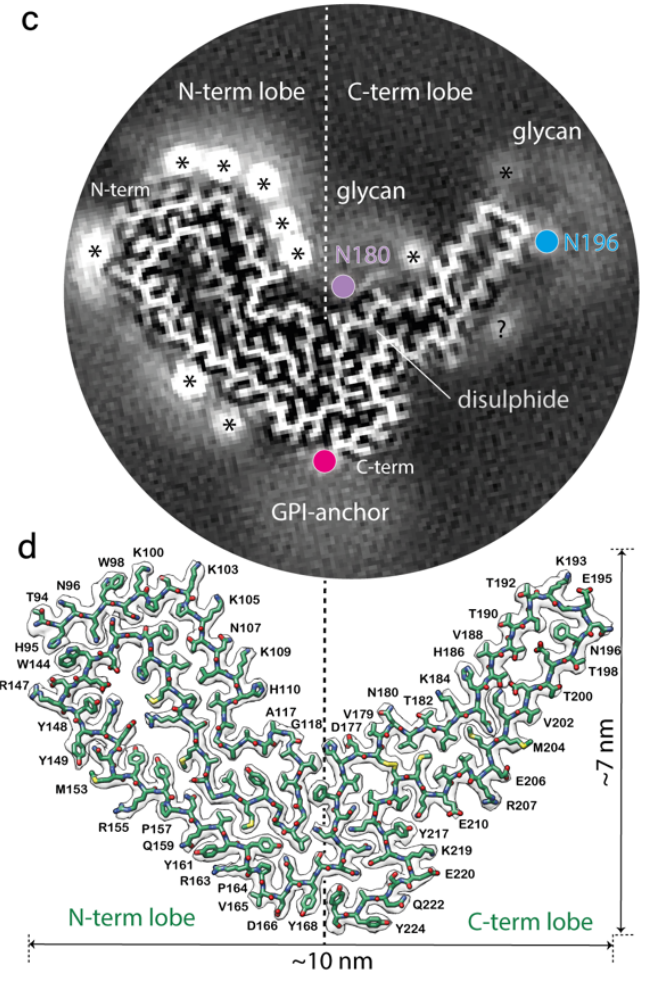
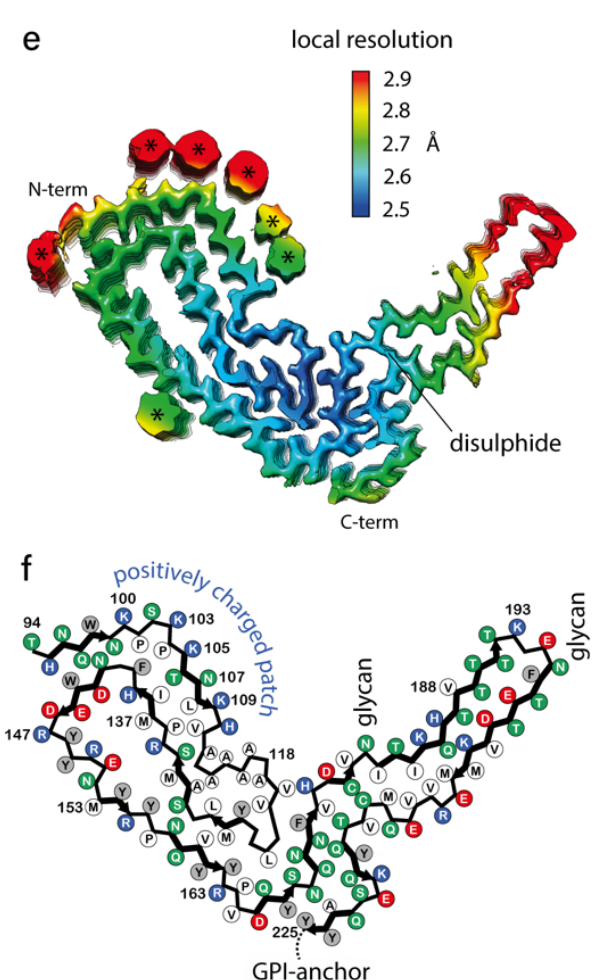
Fig. 1. RML fibril morphologies and 3D reconstruction and atomic model of the RML protofilament core. a) Selected cryo-EM images (300 kV FEI Krios G3i, K3 camera) showing examples of single RML protofilaments (s) and paired protofilaments $(p)$ with approximate measurements of widths and crossover distances. Black arrowheads, partly intertwined protofilaments; white arrowheads, 'notched' paired protofilaments. Pie chart, quantification of paired protofilaments in 6000 micrographs. b) Rendered cryo-EM map (isosurface) view of both sides of a helical crossover, with annotated locations of N-linked glycans and the GPI-anchor. c) Crosssection (as indicated in (b)) of the unrendered map (pixel size: $1.067 \AA$ ) showing the protein core and the nonprotein extra densities, with annotations coloured as in (b); ${ }^{*}$, likely locations of phosphotungstate polyanions (cages) near positively charged residues; ?, unassigned density. See also Extended Data Fig. 4b. d) Protein-only density of a single amyloid rung with the fitted atomic model of the mouse PrP chain shown with sticks and coloured by heteroatom: C, green; N, blue; O, red; S, yellow. e) Local resolution of the map calculated with Relion 3.1 LocRes. ${ }^{*}$, as in (c). f) Diagram of the PrP subunit. Positions of amino acid side chains are indicated with circles (positively charged, blue; negatively charged, red; neutral, green; hydrophobic, white; aromatic, grey) on either side of the backbone (black line). $\beta$-strands are indicated with thick black arrow-headed lines.

The RML protofilament has a PIRIBS structure with outwardly projecting $\mathbf{N}$-linked glycans and GPI-anchor. We determined a $2.7 \AA$ structure of the single RML protofilament and de novo built and refined an atomic model in the cryo-EM density based on mouse PrP sequence (residues T94-Y225) (Fig. 1b-d, Table 1 and Extended Data Fig. 3-5). Similar to fibrils from the hamster $263 \mathrm{~K}$ prion $\operatorname{strain}^{49}$, the RML protofilament displays a PIRIBS amyloid structure, with a single PrP chain contributing each rung or 'rib' to the resultant helical ribbon, with a $4.82 \AA$ A spacing between the rungs, a left-handed helical twist of $-0.64^{\circ}$ and a crossover distance of approx. $1344 \AA$ (Fig. 1b-f). The PK-resistant core has overall dimensions of approx. $10 \times 7 \mathrm{~nm}$ (Fig. 1d) and can be divided into a double-hairpin Nterminal lobe and a single-hairpin C-terminal disulphide-stapled lobe (Fig $1 \mathrm{c}$ and d). The extra (non-protein) densities in the $\mathrm{N}$-terminal lobe are consistent with phosphotungstate polyanions ([$\left[\mathrm{PW}_{11} \mathrm{O}_{39}\right]^{7-}$ at $\left.\mathrm{pH} 7.8\right)$ used to facilitate prion purification ${ }^{54,57,58}$ that form cagelike Keggin structures ${ }^{58,59}$. These bind to solvent-exposed strings of positively-charged residues on the surface of the protofilaments (Fig. 1b, c and e, Extended Data Fig. 4). Extra densities in the C-terminal lobe are seen at the positions of N180- and N196-linked glycans and the flexible GPI-anchor at the C-terminus (Fig. 1C). 
Table 1. Cryo-EM data collection, refinement and validation statistics

\begin{tabular}{|c|c|}
\hline & $\begin{array}{l}\text { RML } \\
(\text { EMDB-xxxx) } \\
\text { (PDB } x x x x)\end{array}$ \\
\hline \multicolumn{2}{|l|}{ Data collection and processing } \\
\hline Magnification & $81,000 x$ \\
\hline Voltage (kV) & 300 \\
\hline Electron exposure $\left(\mathrm{e}-/ \AA^{2}\right)$ & 49 \\
\hline Defocus range ( $\mu \mathrm{m})$ & from -3.0 to -1.5 \\
\hline Pixel size (Å) & 1.067 \\
\hline Symmetry imposed & $\mathrm{C} 1$ \\
\hline Initial particle images (no.) & 771,499 \\
\hline Final particle images (no.) & 119,390 \\
\hline Map resolution $(\AA ̊)$ & 2.7 \\
\hline FSC threshold & 0.143 \\
\hline Map resolution range $(\AA ̊)$ & $2.5-3$ \\
\hline \multicolumn{2}{|l|}{ Refinement } \\
\hline Initial model used (PDB code) & de novo \\
\hline Model resolution $(\AA ̊)$ & 2.7 \\
\hline FSC threshold & 0.143 \\
\hline Model resolution range $(\AA ̊)$ & $2.7-50$ \\
\hline Map sharpening $B$ factor $\left(\AA^{2}\right)$ & -36.9 \\
\hline \multicolumn{2}{|l|}{ Model composition } \\
\hline Non-hydrogen atoms & 6255 \\
\hline Protein residues & 396 \\
\hline Ligands & none \\
\hline \multicolumn{2}{|l|}{$B$ factors $\left(\AA^{2}\right)$} \\
\hline Protein & $31.38-66.09$ \\
\hline \multicolumn{2}{|l|}{ r.m.s. deviations } \\
\hline Bond lengths $(\AA)$ & 0.005 \\
\hline Bond angles $\left({ }^{\circ}\right)$ & 0.693 \\
\hline \multicolumn{2}{|l|}{ Validation } \\
\hline MolProbity score & 1.56 \\
\hline Clashscore & 2.72 \\
\hline Poor rotamers (\%) & 0 \\
\hline \multicolumn{2}{|l|}{ Ramachandran plot } \\
\hline Favored (\%) & 91.54 \\
\hline Allowed (\%) & 8.46 \\
\hline Disallowed (\%) & 0 \\
\hline
\end{tabular}


a
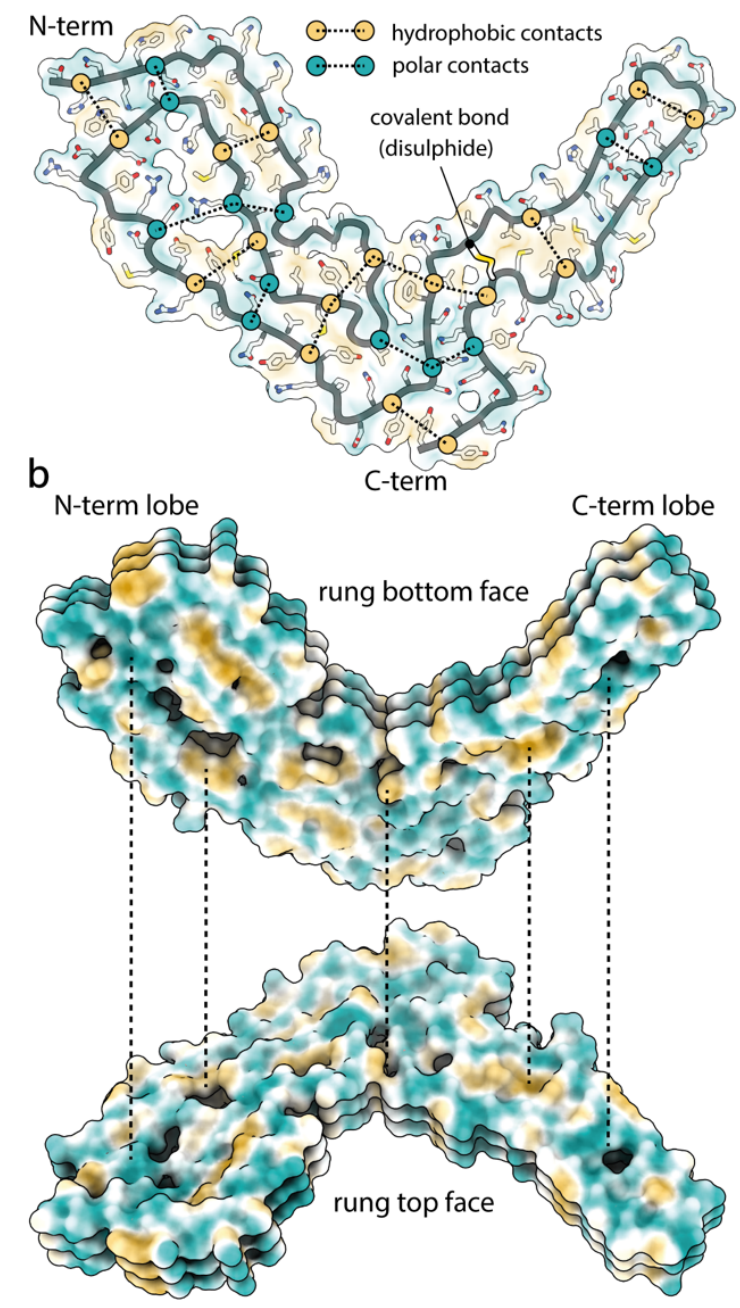

C

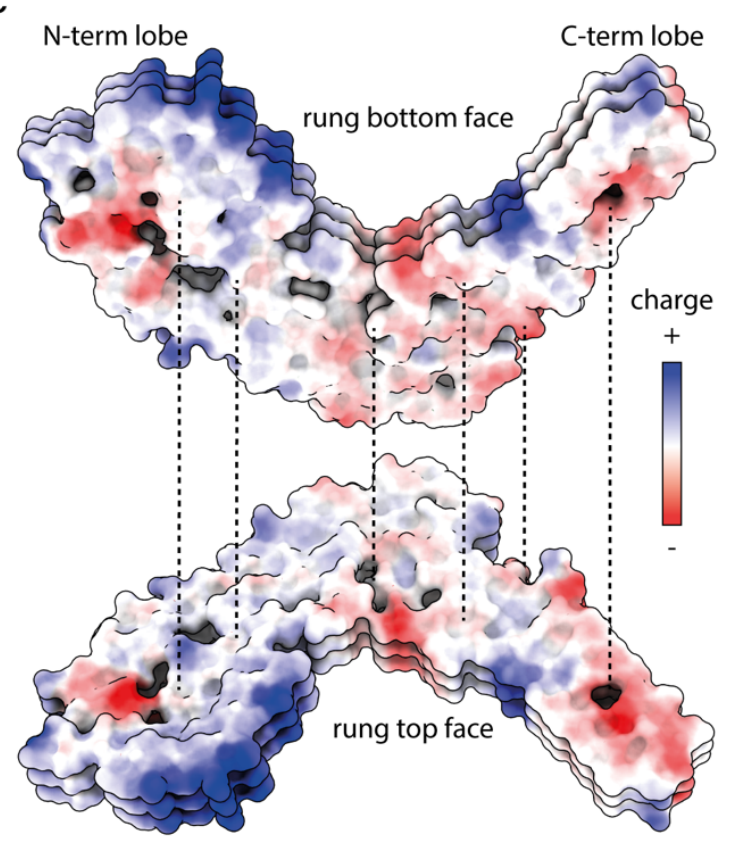

d

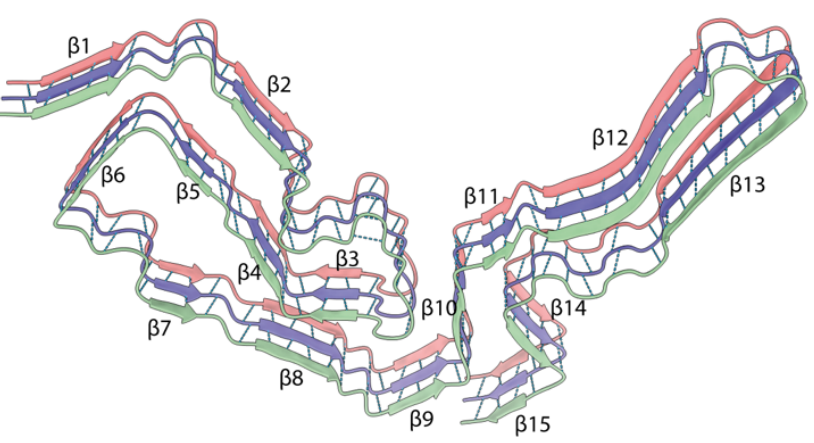

Fig. 2. Intra- and inter-chain interactions stabilising the RML prion fibril. a) Schematic depiction of the alternating polar and non-polar lateral contacts that stabilise a single PrP monomer in the fibril. Transparent surface representation is coloured by hydrophobicity (hydrophobic, yellow; hydrophilic, teal). Protein backbone is shown with cartoon (licorice) representation and amino acid side chains as white sticks coloured by heteroatom ( $O$, red; $N$, blue; $S$, yellow). b) Butterfly view of the top and bottom surface of each rung, coloured as in (a). Dotted lines indicate longitudinally connecting regions in the assembly. c) Butterfly view of charge distribution in the assembly. Dotted lines as in (b). d) Ribbon representation of 3 amyloid rungs, with indicated $\beta$-sheets and inter-chain hydrogen bonds (dotted lines).

Intra- and inter-molecular interactions in RML protofilaments. Alternating polar and hydrophobic intra-chain interactions stabilise the conformation of the PrP chain in each amyloid rung (Fig. 2a) and form hydrophobic and hydrophilic columns along the fibril (Fig. 2b), similar to the way in which charges distribute along the fibril (Fig. 2c). These longitudinal interactions likely contribute to the extraordinary stability of the assembly and may play an important role in templating PrP misfolding, refolding into the prion strainspecific conformation and resistance to host clearance mechanisms. The other major intermolecular interactions are the typical hydrogen bonds in the PIRIBS arrangement (Fig. $2 \mathrm{~d}$ ). Our model suggests that there are 15 inter-chain $\beta$-sheets in the RML fibril (Fig. $2 \mathrm{~d}$ ). 
bioRxiv preprint doi: https://doi.org/10.1101/2021.12.13.472424; this version posted December 14, 2021. The copyright holder for this preprint (which was not certified by peer review) is the author/funder, who has granted bioRxiv a license to display the preprint in perpetuity. It is made available under aCC-BY-NC 4.0 International license.

$\mathrm{N}$ - and C-terminal lobes of the PrP subunits in the RML protofilament are staggered. Considering longitudinal stacking of PrP monomers, the $\mathrm{N}$ - and C-terminal lobes of the chain are staggered along the amyloid fibril axis, so that each $\mathrm{N}$-terminal lobe is co-planar with the C-terminal lobe of the consecutive rung (Fig. 3). Hydrophobic interactions between the Nterminal lobe's V120 and the C-terminal lobe's F174 and H176 mediate the contact between the staggered lobes (Fig. 3). Similar, but more pronounced staggering was reported for fibrils from the $263 \mathrm{~K}$ prion hamster $\operatorname{strain}^{49}$ (Fig. 4a, bottom panel), in which each $\mathrm{N}$ terminal lobe contacts the $\mathrm{C}$-terminal lobe of the second consecutive rung.



Fig. 3. Staggering of the $\mathrm{N}$ - and $\mathrm{C}$ terminal lobes in the RML prion fibril. Top, cryo-EM density (MAP) and solvent-excluded surface (MODEL) of three rungs, with indicated side chains that connect to form the inter-lobe contacts. The F174 and $\mathrm{H} 176$ residues are better visible in the MAP and MODEL views, respectively. Bottom, magnified view of the staggered interactions (hydrophobic contacts) shown with transparent surface, main chain as ribbon and selected interacting residues as sticks. 
Comparison of mouse RML protofilaments with hamster 263K fibrils. When the two PrP conformations are aligned on the first two $\beta$-strands, the N-terminal lobes of the two strains look relatively similar (Fig. 4a), although the first hairpin structure in the RML conformation does not resemble a 'Greek key' - as it was named in the $263 \mathrm{~K}$ fibril conformation ${ }^{49}$ - and the N-terminal lobe of the $263 \mathrm{~K}$ fibril appears less tightly packed than that of RML (Fig. $4 \mathrm{~b}$, see holes in the solvent-accessible surface), whereas the C-terminal lobes are markedly divergent (Fig. 4a). The distance between the first glycosylation site (N180/181 in the mouse/hamster sequence) is $\sim 7 \AA$ and it increases to $\sim 34 \AA$ for the second glycosylation site (N196/197 in the mouse/hamster sequence). Then the two folds become closer again between residues R207/208-Q216/217 (mouse/hamster numbering) and diverge again at their C-termini. The last residues of the ordered protein cores of RML and $263 \mathrm{~K}$ fibrils are $\sim 36 \AA$ apart (Fig. 4a, top panel). The side view of the alignment (Fig. 4a, bottom panel) reveals the more pronounced inter-lobe stagger observed in the $263 \mathrm{~K}$ fibril. This difference may be linked to the tighter helical twist of the 263K fibril (crossover distance of $\sim 100 \mathrm{~nm}^{49}$ ) compared to that of the RML protofilament (crossover distance of $\sim 135 \mathrm{~nm}$ ).

The C-terminus and tip of the C-terminal lobe is where the two structures deviate the most. This tip appears rigid in RML protofilaments, likely due to relatively tight interactions stabilising the corresponding hairpin structure (Figs. 2a and d), whereas in the $263 \mathrm{~K}$ fibril reconstruction, the density for the three amino acid residues at that tip (K194E196) is missing (Fig. 4b), which indicates local flexibility or disorder. This flexibility is likely a consequence of the divergent fold in the 263K fibril C-terminal lobe compared to RML protofilaments. The C-terminal lobe of the RML protofilament continues to bind along the opposing strand in the hairpin structure, resulting in the C-terminal lobe being a single hairpin, while in the $263 \mathrm{~K}$ fibril the chain pivots at residue $\mathrm{Y} 218$ to swing in the opposite direction and fold back on itself, forming a double hairpin structure (Fig. 4a-c). There, at the base of that second hairpin, residue Y226 interacts with 1203 and 1205 , creating a gap in the first hairpin, which in turn destabilises its tip (which is the tip of the C-terminal lobe) (Fig. 4c, bottom panel). The difference in the conformation of the $\mathrm{C}$-terminal lobe between the two fibrils corresponds to the differences in their host PrP sequence (Fig. 4c, top panel). These differences result in a narrower groove between the $\mathrm{N}$ - and $\mathrm{C}$-terminal lobes in the RML PrP protofilament than in the $263 \mathrm{~K}$ hamster PrP fibril (Fig. 4b). The inter-lobe angle in our RML protofilament structure is similar to that of the recently published low-resolution crosssection of fibrils from RML prion-infected transgenic mice expressing GPI-anchorless PrP ${ }^{49}$. 

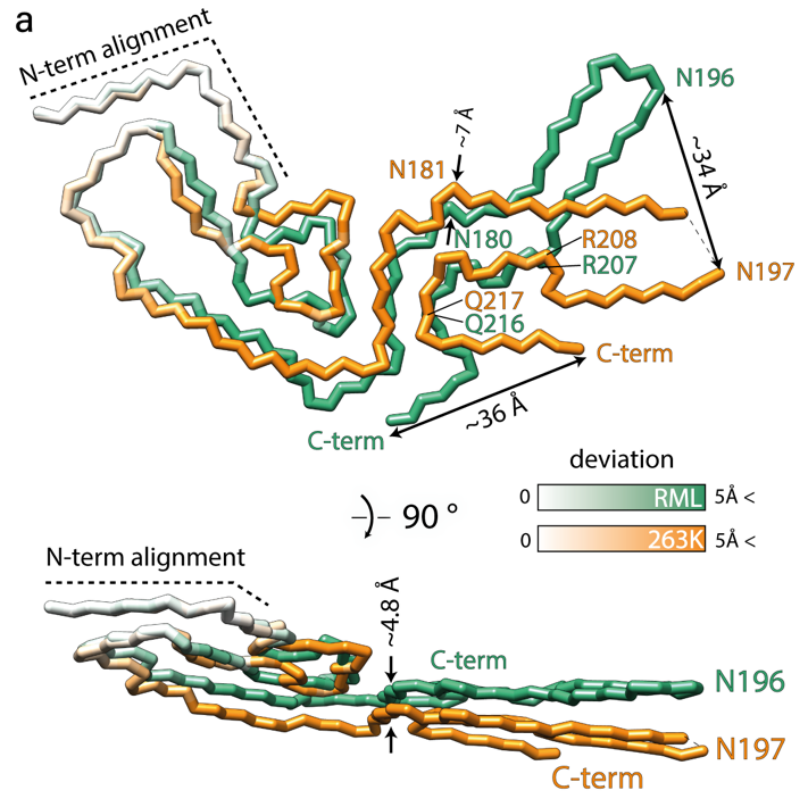

b

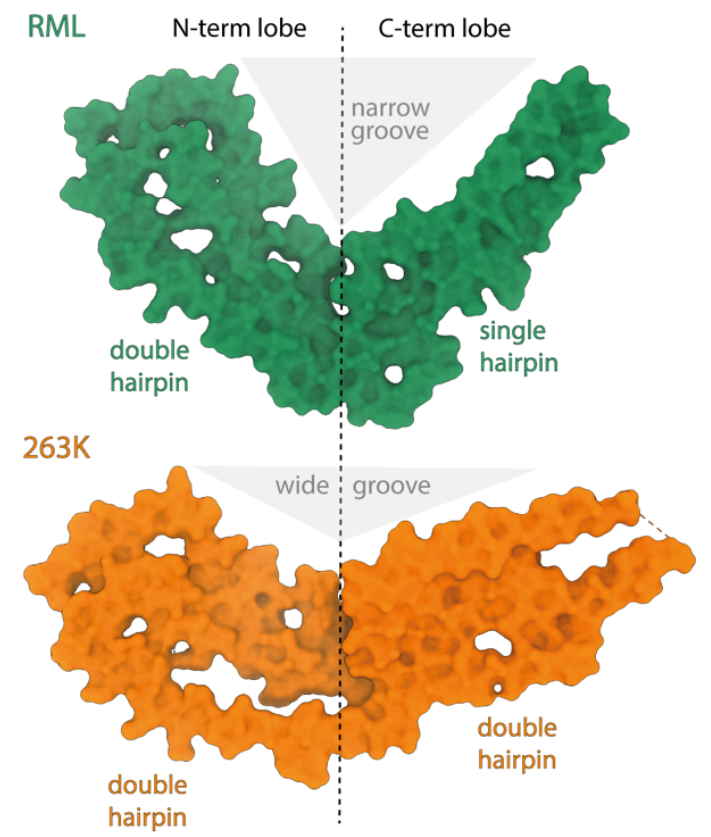

C
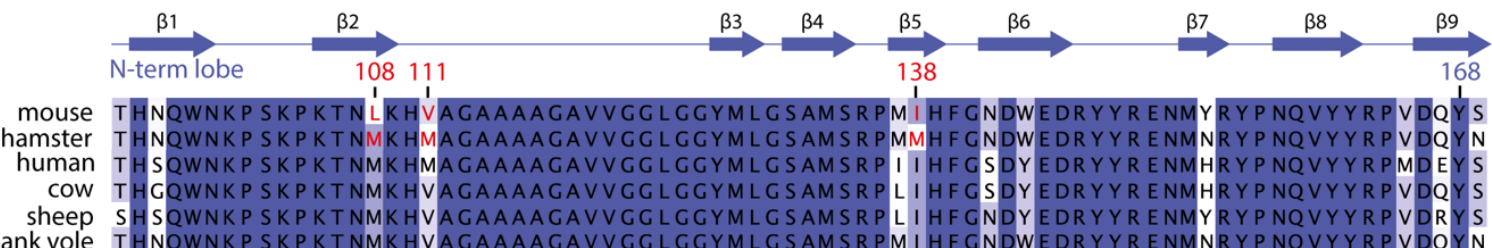

bank vole THNOWNKPSKPKTNM
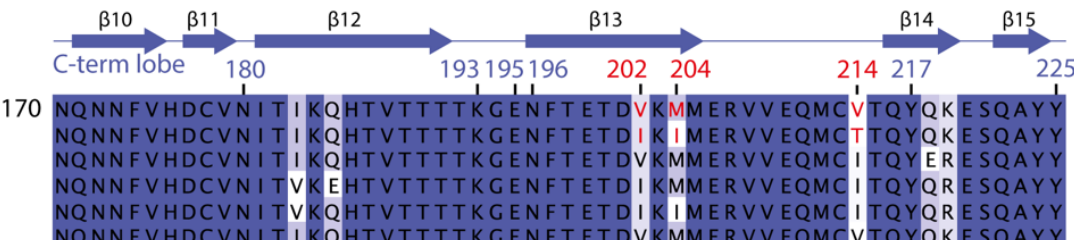

NQNNFVHDCVN I TVKQHTVTTTTKGENFTETDIKIMERVVEQMCI TQYQRESQAYY

NQNNFVHDCVN I TIKQHTVTTTTKGENFTETDVKMMER VVEQMCVTQYQKESQAYY

RML

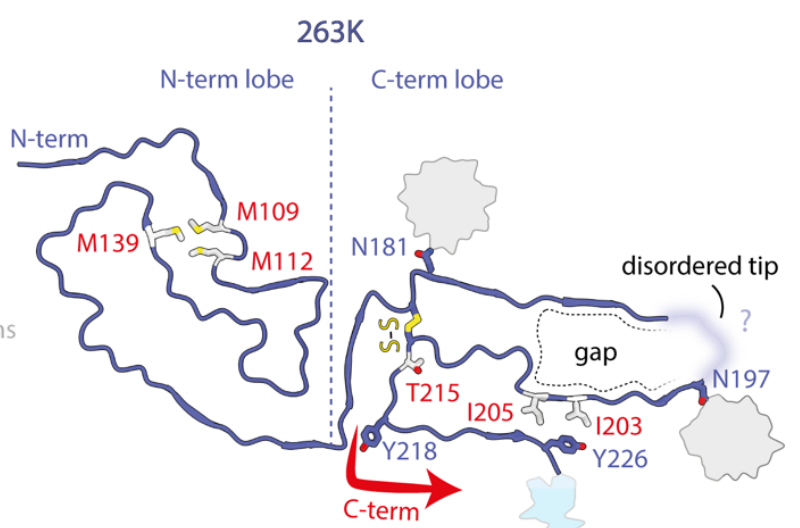

GPI-anchors

Fig. 4. Comparison of PrP conformation in the RML and the $263 \mathrm{~K}$ prion fibrils. a) Polypeptide backbone superposition on the first two $\beta$-strands ( $N$-term alignment; secondary structure not shown) of single PrP monomers from the two different strains, coloured by their deviation in distance. b) Surface models showing internal gaps and the divergent angles between the $\mathrm{N}$ - and C-terminal lobes. c) Top, multiple PrP sequence 
alignment coloured by conservation and annotated by mouse RML PrP sequence numbering and secondary structure. Mouse vs. hamster amino acid substitutions (AAS) that underpin distinct conformations of RML and 263K fibrils are highlighted in red. Bottom, mapping of the selected AAS onto cartoon structures of RML (this study) and 263K (pdb code: 7LNA) fibrils (sticks coloured white and by heteroatom: O, red; S, yellow). Selected conserved residues, including those involved in distinct interactions due to divergent PrP folds are shown with sticks coloured as main chain and by heteroatom ( $\mathrm{N}$, blue; $\mathrm{O}$, red; S, yellow). Red arrows indicate different folds of the C-termini, which result in divergent tips of the C-terminal lobes.

\section{Discussion}

In the present study we have determined a $2.7 \AA$ structure of single protofilaments in highly purified preparations of infectious mouse RML prions and compared this with the recently published cryo-EM structure of fibrils in highly purified preparations of hamster $263 \mathrm{~K}$ prions ${ }^{49}$. Both fibrils have a PIRIBS conformation with a PrP subunit forming each rung of the fibril, and protease resistant cores that correspond to the sequences expected from the strain-specific signature PrP 27-30 truncated PrPSc banding patterns seen on western blots. The fold in the core of both fibrils creates distinct $\mathrm{N}$ - and $\mathrm{C}$-terminal lobes in each PrP monomer thereby generating a broadly similar overall architecture in which the $\mathrm{N}$-linked glycans and GPI anchor project from the C-terminal lobe. While the architectures of ex vivo mouse RML and hamster $263 \mathrm{~K}$ fibrils are clearly similar, they are notably distinct from the recently reported cryo-EM structures of recombinant PrP amyloid ${ }^{47,48,50}$ and the $\beta$-solenoid structure postulated for ex vivo mouse PrP amyloid purified from the brain of RML-prion infected transgenic mice expressing GPI-anchorless PrP60.

Despite the overall structural similarity between mouse RML and hamster $263 \mathrm{~K}$ fibrils there are pronounced differences in the fold of the C-terminal lobes which may be attributable to differences in PrP primary structure and/or represent distinct conformational templating by divergent prion strains. To directly observe strain-specific prion conformations on the same PrP sequence, high resolution cryo-EM structures of fibrils from other mouse- or hamsteradapted prion strains are required.

Authentic prion structures should account for the mechanism by which high-fidelity selection of PrP glycoforms is achieved during prion assembly ${ }^{2,3,38}$. The glycoform ratios of PrP in mouse RML fibrils and hamster 263K fibrils are very different, with marked predominance of diglycosylated $\mathrm{PrP}$ in the $263 \mathrm{~K}$ fibril $^{49}$ and predominance of monoglycosylated PrP's in the RML fibril ${ }^{54}$ (Extended Data Fig. 1). Although in silico modelling suggests no obvious steric hindrance for accommodating even solely di-glycosylated PrP chains into a cross- $\beta$ amyloid structure ${ }^{61}$, the degree of helical twist and the size of the cleft between the $\mathrm{N}$-and $\mathrm{C}$-terminal lobes in which residue N180/N181 (mouse/hamster numbering) is contained may favour certain PrP glycoforms over others. Notably, the $263 \mathrm{~K}$ fibril has a much wider groove between the $\mathrm{N}$ - and C-terminal lobes than RML fibrils (Fig. 4b), which may sterically permit more glycan occupancy at N181. Moreover, the 263K fibril has a greater helical twist than RML fibrils $\left(100 \mathrm{~nm}^{49}\right.$ versus $135 \mathrm{~nm}$ crossover distance, respectively), which may help to displace successive glycans and thereby accommodate a higher glycan occupancy at both N181 and N197 sites. These structural differences may favour incorporation of di-glycosylated PrP monomers (which are the most abundant PrPC glycoforms) into the $263 \mathrm{~K}$ fibril over the RML fibril. 
An alternative mechanism for generating strain-specific PrP glycoform ratios is the propagation of paired protofilaments in which the architecture of the pairs sterically limits the space available for glycan occupancy as monomers assemble into each protofilament. Within such architectures the glycans themselves may also interact with one another and contribute to the overall stability of the assembly and to the ability of prions to evade host defences. Our observation of paired protofilaments in the purified RML prion samples may suggest such a mechanism and paired protofilaments have also recently been reported in purified samples from L-type BSE-prion-infected transgenic mouse brain ${ }^{62}$. Due to an insufficient number of segments we could not obtain a conclusive 3D reconstruction of the paired protofilaments assembly in this study. It will now be critical to determine the paired structure and whether the single protofilaments we observe in our samples (i) originate from pairs which are the replicating species, (ii) replicate independently and then pair, or (iii) coexist with pairs as two independent seed architectures.

\section{Methods}

\section{Research governance}

Frozen brains from mice with clinical prion disease were used to generate purified prion samples. These brain samples were generated by us as part of a previous study ${ }^{54}$ in which work with animals was performed in accordance with licences approved and granted by the UK Home Office (Project Licences 70/6454 and 70/7274) and conformed to University College London institutional and ARRIVE guidelines. All experimental protocols were approved by the Local Research Ethics Committee of UCL Queen Square Institute of Neurology/National Hospital for Neurology and Neurosurgery. Prion purification, cell based prion bioassay and preparation of cryo-EM grids was conducted at UCL in microbiological containment level 3 or level 2 facilities with strict adherence to safety protocols. Work with infectious prion samples at Birkbeck College London was performed using dedicated sample holders and equipment with strict adherence to safety procedures and local risk assessment. Prion samples were transported between laboratories in packaging conforming to UN 3373 Biological Substance, Category B specifications.

\section{Preparation of purified RML prion rods}

Prion-infected brain homogenate was prepared by homogenizing 200 brains from CD-1 mice terminally-infected with the RML prion strain in Dulbecco's phosphate buffered saline (DPBS; Gibco) to produce a pool of 1 litre $10 \%$ (w/v) RML brain homogenate (designated 117700) using methods described previously ${ }^{54}$.

Purification of RML prion rods was performed as described previously ${ }^{54}$ with minor modifications. Initial protease digestion was performed using proteinase $\mathrm{K}$ at a working concentration of $50 \mu \mathrm{g} \mathrm{ml}^{-1}$ in the place of pronase E. Samples were incubated for 30 mins at $37^{\circ} \mathrm{C}$ with constant agitation at $800 \mathrm{rpm}$, after which digestion was terminated by addition of $100 \mathrm{mM}$ 4-(2-Aminoethyl) benzenesulfonyl fluoride hydrochloride (AEBSF) to give $2 \mathrm{mM}$ final concentration in the sample. The purification protocol was then followed as described until the $\mathrm{P} 2$ stage $(\mathrm{P}=$ pellet), whereupon the final two wash steps were replaced with a single wash in $1.0 \mathrm{ml}$ of $5 \mathrm{mM}$ sodium phosphate buffer $(\mathrm{pH} 7.4)$ containing $0.1 \%$ 
$(\mathrm{w} / \mathrm{v})$ sarkosyl (Calbiochem) and $0.3 \%(\mathrm{w} / \mathrm{v})$ sodium phosphotungstate, in order to avoid unnecessary aggregation of the purified rods arising from repeated rounds of centrifugation. Final P3 samples were typically resuspended to a concentration of $120 \mathrm{X}$ relative to the starting volume of $10 \%(\mathrm{w} / \mathrm{v})$ brain homogenate for loading on to EM grids.

Prion infectivity of brain homogenates or purified samples was measured in the Scrapie Cell End Point Assay (SCEPA) ${ }^{56}$ using PK1/2 cells. Every experiment included concomitant assay of a serial dilution of RML prions of known prion titre determined from rodent bioassay to produce a standard curve that unknown samples were calibrated against. $10 \%(\mathrm{w} / \mathrm{v}) \mathrm{RML}$ brain homogenate 16200 was used as the standard and reported a prion titre of $10^{7.3+0.5}$ (mean + s.d.) intracerebral $L_{50}$ units $/ \mathrm{ml}$ when endpoint titrated six times in $\mathrm{Tg} 20$ mice that overexpress mouse PrP on a Prnp ${ }^{\circ / o}$ background, corresponding to $10^{7.7} \mathrm{TCIU} / \mathrm{ml}$ in PK1/2 cells ${ }^{54}$. PrP concentrations in purified samples were measured by ELISA as described previously ${ }^{54}$.

\section{SDS-PAGE, silver staining and western blotting}

Samples were prepared for SDS-PAGE using NuPage 4X LDS buffer and 10X Reducing Agent (Thermo Fisher) according to the manufacturer's instructions followed by immediate transfer to a $100{ }^{\circ} \mathrm{C}$ heating block for $10 \mathrm{~min}$. Electrophoresis was performed on NuPage 12 $\%$ Bis-Tris protein gels (Thermo Fisher), run for $60 \mathrm{~min}$ at $200 \mathrm{~V}$, prior to electroblotting to Immobilon P membrane (Millipore) for $16 \mathrm{~h}$ at $15 \mathrm{~V}$. Membranes were blocked in $1 \mathrm{X}$ PBS (prepared from 10X concentrate; VWR International) containing $0.05 \%(\mathrm{v} / \mathrm{v})$ Tween 20 (PBST) and 5\% (w/v) non-fat dried skimmed milk powder and then probed with $0.2 \mu \mathrm{g} / \mathrm{ml}$ anti-PrP monoclonal antibody ICSM35 (D-Gen Ltd) in PBST for at least $1 \mathrm{~h}$. After washing (1 h with PBST) the membranes were probed with a 1:10,000 dilution of alkaline-phosphataseconjugated goat anti-mouse IgG secondary antibody (Sigma-Aldrich) in PBST. After washing ( $1 \mathrm{~h}$ with PBST and $2 \times 5 \mathrm{~min}$ with $20 \mathrm{mM}$ Tris $\mathrm{pH} 9.8$ containing $1 \mathrm{mM} \mathrm{MgCl}$ ) blots were incubated for $5 \mathrm{~min}$ in chemiluminescent substrate (CDP-Star; Tropix Inc) and visualized on Biomax MR film (Carestream). SDS-PAGE gels (prepared as above) were silver stained using the Pierce Silver Stain Kit (Thermo Fisher) according to the manufacturer's instructions. Gels were photographed on a light box using a Nikon Coolpix P6000 digital camera. Typical sample loadings for western blotting or silver staining correspond to purified material derived from $10 \mu \mathrm{l}$ or $100 \mu \mathrm{l}$ of $10 \%(\mathrm{w} / \mathrm{v})$ RML prion-infected brain homogenate per lane, respectively.

\section{RML sample preparation for cryo-EM}

RML prion rods purified from $2.4 \mathrm{ml} 10 \%(\mathrm{w} / \mathrm{v})$ RML-infected brain homogenate were resuspended from the $\mathrm{P} 3$ pellet (see above) in 20-30 $\mu \mathrm{l} 5 \mathrm{mM}$ sodium phosphate buffer $(\mathrm{pH}$ 7.4) supplemented with $0.1 \%(\mathrm{w} / \mathrm{v})$ sarkosyl and $4 \mu \mathrm{l}$ of the suspension was applied directly to a glow-discharged C-flat ${ }^{\mathrm{TM}}$ Holey Carbon CF-2/2-4C Cu 400 mesh cryo-EM grid (Electron Microscopy Sciences) in the chamber of the Leica GP2 plunging robot. The chamber was set to $20^{\circ} \mathrm{C}$ and $50 \%$ humidity. After $10 \mathrm{~s}$ incubation, the grids were blotted for $3 \mathrm{~s}$ (with an additional $2 \mathrm{~mm}$ push) and plunge-frozen in liquid ethane maintained at $-183^{\circ} \mathrm{C}$.

\section{Cryo-EM data collection}

Cryo-micrographs were acquired at Birkbeck College London, on a $300 \mathrm{kV}$ Krios G3i microscope (FEl/Thermo Fisher) with a post-GIF (20 eV slit) K3 detector (Gatan) operated in 
super-resolution mode. The magnified pixel size was $0.5335 \AA$. The dose rate was 16.37 e$/ \AA^{2} /$ s during 3-s exposures, resulting in the total dose of 49 e- $/ \AA^{2}$ on the specimen. The exposures were collected automatically at 4 shots per grid hole, with fast acquisition ( 240 images/hr), using the EPU software (FEI/Thermo Fisher), at defocus ranging from -3.0 to 1.5 , and fractionated into 50 movie frames.

\section{Cryo-EM image processing and 3D reconstruction}

All image processing except particle picking was done within the framework of Relion $3.1^{41}$. We used Relion's implementation of the MotionCor2 algorithm to align movie frames. The images were $2 x$ binned in Fourier space during the frame alignment, resulting in the final pixel size of $1.067 \AA^{2}$ in the drift-corrected sums. The contrast transfer function (CTF) parameters were estimated with Gctf ${ }^{63}$. We then picked particles (fibril segments) using the deep learning package crYOLO $^{64}$ trained on 100 example micrographs. The picking was accurate and avoided crowded regions with overlapping or clumped rods, fibrils on carbon support and fibrillar bundles, as illustrated in Extended Data Fig. 3. We imported the coordinates into Relion and extracted images of prion rod segments of different box sizes (ranging from 1024 to 384 pixels) to perform reference-free 2D classification. Optimal 2D class averages and segments were selected for further processing and used to de novo generate an initial 3D reference with relion_helix_inimodel2d programme ${ }^{41}$, using an estimated rise of $4.75 \AA$ and helical twist according to the observed crossover distances of the filaments in the 2D class averages. After $3 \mathrm{D}$ classification and $3 \mathrm{D}$ auto-refinement, we obtained a 3D reconstruction of the RML fibril at $3.0 \AA$ resolution in a 384-pixel cube. Subsequent Bayesian polishing ${ }^{65}$ and CTF refinement ${ }^{66}$ were performed to further improve the resolution of the reconstruction to $2.7 \AA$, according to 0.143 FSC cut-off criterion (Extended Data Fig. 5a).The final 3D map was sharpened with a generous, soft-edged solvent mask at $10 \%$ of the height of the box using the computed B-factor value of $-36.9 \AA^{2}$. The sharpened map was used for the subsequent atomic model building and refinement. The local resolution calculation was performed by LocRes in Relion 3.1 with solvent mask over the entire map.

\section{Atomic model building and refinement}

A single subunit repeat was extracted in UCSF Chimera ${ }^{67}$ for the initial de novo model building in $\mathrm{Coot}^{68}$. The initial atomic model was then copied and fitted into 3 consecutive subunits in the map and the map was zoned around the atomic coordinates in UCSF Chimera ${ }^{67}$. The 3-rung map and model were placed in a new unit cell with P1 space group for subsequent model refinement using default settings in phenix.real_space_refine ${ }^{69}$ and REFMAC5 $5^{70}$ with non-crystallographic symmetry (NCS) group definitions constraining the helical subunit repeat. Model geometry was evaluated using the MolProbity server ${ }^{71}$ (http://molprobity.biochem.duke.edu/) after each round of refinement, and problematic or poorly fitting regions in the model were manually adjusted using $\operatorname{Coot}^{68}$ and Isolde ${ }^{72}$ (within Chimera $X^{73}$ ). This process was repeated until a satisfactory level of model:map agreement with acceptable model stereochemistry was achieved (Table 1).

\section{Negative-stain EM}

RML prion rods purified from $2.4 \mathrm{ml} 10 \%(\mathrm{w} / \mathrm{v}) \mathrm{RML}$-infected brain homogenate were resuspended from the P3 pellet (see above) in $40 \mu \mathrm{l} 20 \mathrm{mM}$ Tris- $\mathrm{HCl} \mathrm{pH} \mathrm{7.4,} 150 \mathrm{mM} \mathrm{NaCl}$ (TBS) containing $0.1 \%(\mathrm{w} / \mathrm{v})$ sarkosyl and deposited on glow-discharged EM grids with a 
continuous carbon film (Agar). The grids were briefly blotted and washed with TBS before staining with $2 \%$ solution of NANO-W ${ }^{\mathrm{TM}}$ stain (Nanoprobes). After $\sim 1 \mathrm{~s}$ exposure to the stain solution, the grids were blotted again and air-dried. The negatively stained grids were imaged in the Unit on a $120 \mathrm{kV}$ Talos microscope (FEl/Thermo Fisher) with a $4 \mathrm{k} \times 4 \mathrm{k}$ BMCeta camera.

\section{Structure analyses and presentation}

Analyses and visualisations of the cryo-EM density map and the models compared in this study were done using UCSF Chimera ${ }^{67}$ and ChimeraX ${ }^{73}$.

\section{Acknowledgements}

This work was funded by the core award to the MRC Prion Unit from the UK Medical Research Council (MC_U12316055 and MC_UU_00024/5). EM data collection was supported by grants from the Wellcome Trust (079605/Z/06/Z, 101488/Z/13/Z). We are very grateful to Dr Natasha Lukoyanova and Dr Shu Chen at Birkbeck College for EM support and Damian Johnson, Peter King, Kevin Williams and Kevin Foulger for infrastructure support at UCL. We would like to dedicate this paper to the late Professor Anthony Clarke who founded the protein structure programme at the MRC Prion Unit and who made many fundamental contributions to this field.

\section{Conflict of interests}

J.C. is a Director and J.C. and J.D.F.W. are shareholders of D-Gen Limited, an academic spinout company working in the field of prion disease diagnosis, decontamination, and therapeutics. D-Gen supplied the ICSM35 and ICSM18 antibodies used for western blot and ELISA performed in this study. The other authors declare no potential conflict of interest. 
bioRxiv preprint doi: https://doi.org/10.1101/2021.12.13.472424; this version posted December 14, 2021. The copyright holder for this

preprint (which was not certified by peer review) is the author/funder, who has granted bioRxiv a license to display the preprint in perpetuity. It is made available under aCC-BY-NC 4.0 International license.

\section{Extended Data Figures}

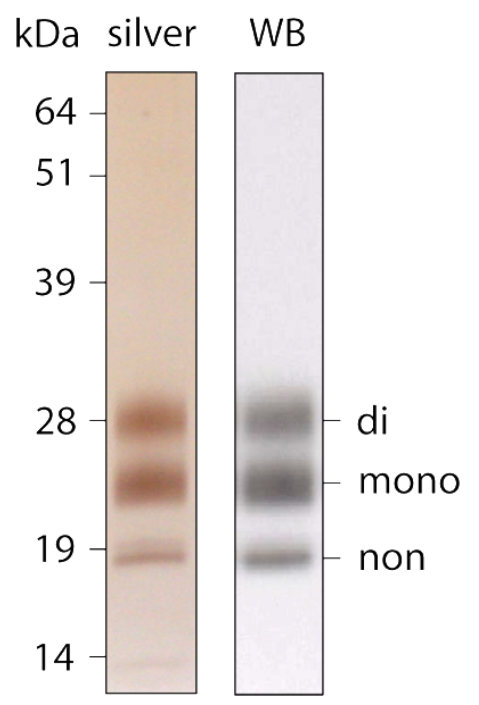

Extended Data Fig. 1. Western blot (WB) and silver-stained SDS-PAGE of purified RML rods. di, mono and non stand for di-, mono- and non-glycosylated PrP bands, respectively. The samples were prepared as described in Methods.

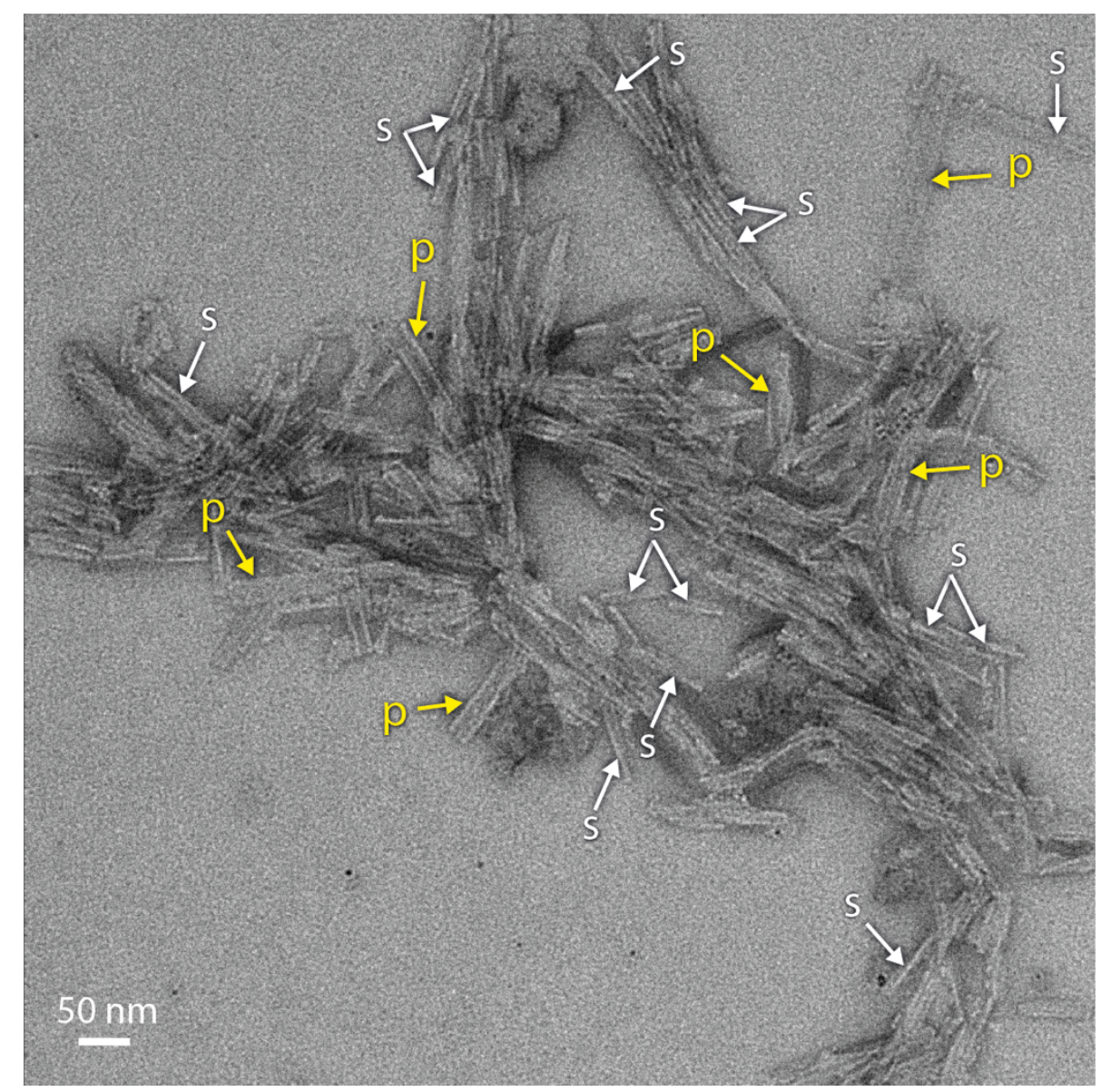

Extended Data Fig. 2. Negative-stain EM of purified RML prion rods. Clear examples of single (s) and paired (p) fibrils are indicated. The aggregated (clumped) fibrils are difficult to interpret or quantify. The rods were stained with $2 \%$ solution of NANO-W ${ }^{\mathrm{TM}}$ stain (Nanoprobes) and imaged on a $120 \mathrm{kV}$ Talos microscope (FEl/Thermo Fisher) with a 4k x 4k BM-Ceta camera. 
bioRxiv preprint doi: https://doi.org/10.1101/2021.12.13.472424; this version posted December 14, 2021. The copyright holder for this preprint (which was not certified by peer review) is the author/funder, who has granted bioRxiv a license to display the preprint in perpetuity. It is made available under aCC-BY-NC 4.0 International license.

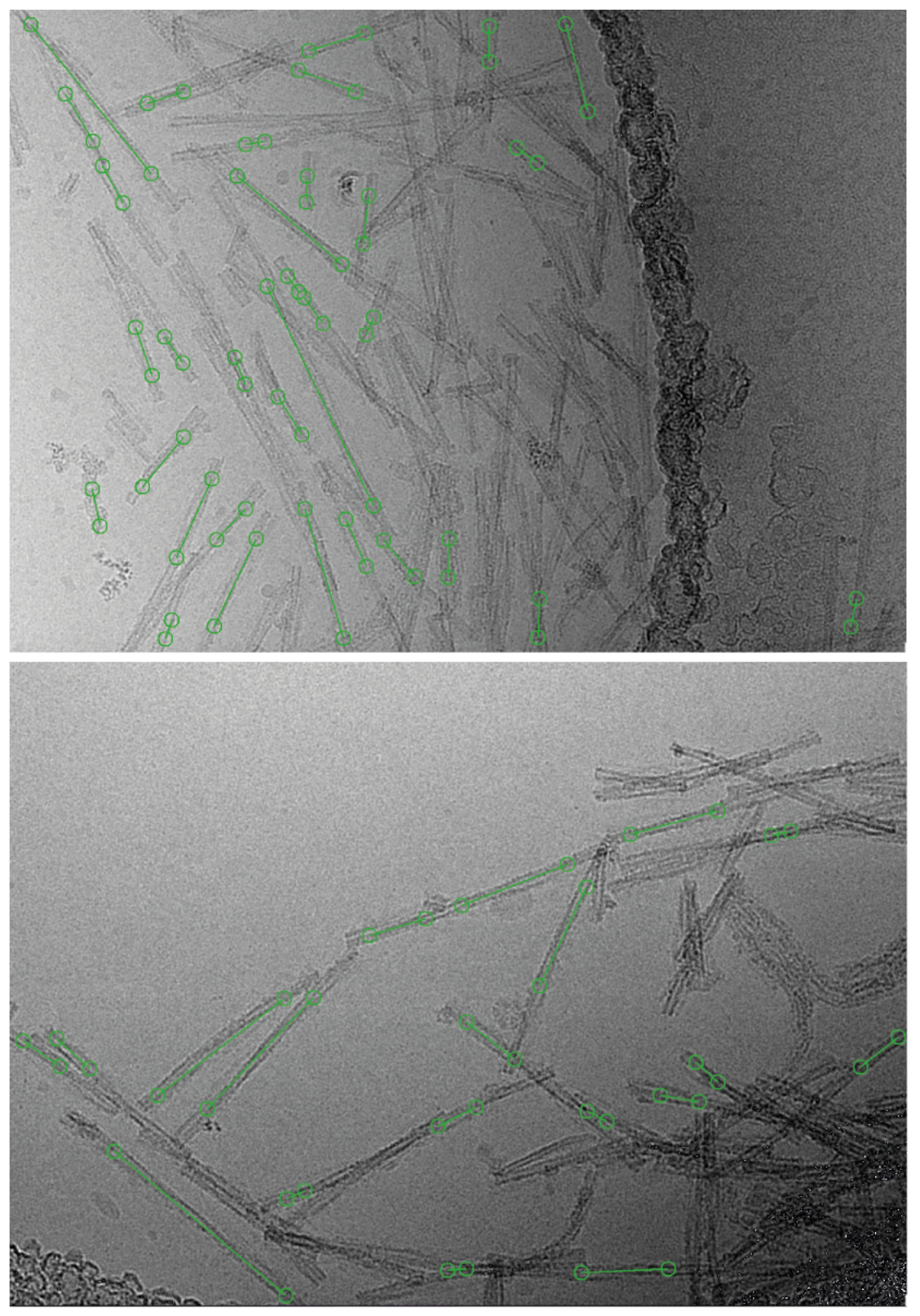

Extended Data Fig. 3. Automated picking of RML rod particles with crYOLO. Shown are example raw cryomicrographs (after movie frame alignment; collected on a Krios G3i microscope with a K3 camera (Gatan) as described in Methods) with example filament picking by crYOLO $^{74}$ (green circles for fibril start-end coordinates connected by a line). The programme mostly picked single fibrils and avoided clumped or bundled fibrils (as trained), but occasionally picked the unwanted narrower views of paired filaments or filaments on carbon support. These erroneously picked particles were excluded from further processing and 3D reconstruction through 2D and 3D classifications. 
bioRxiv preprint doi: https://doi.org/10.1101/2021.12.13.472424; this version posted December 14,2021 . The copyright holder for this

preprint (which was not certified by peer review) is the author/funder, who has granted bioRxiv a license to display the preprint in perpetuity. It is made available under aCC-BY-NC 4.0 International license.



b



Extended Data Fig. 4. 2D classification of RML prion fibrils and extended interpretation of their average cross-section. a) Gallery of representative 2D classes (box size: $384 \times 384$ pixels). b) Single PrP subunit ball and stick model (backbone, grey; side chain atoms: C, grey; O, red; N, blue; S, yellow) superposed on the fibril 3D 
bioRxiv preprint doi: https://doi.org/10.1101/2021.12.13.472424; this version posted December 14,2021 . The copyright holder for this preprint (which was not certified by peer review) is the author/funder, who has granted bioRxiv a license to display the preprint in perpetuity. It is made available under aCC-BY-NC 4.0 International license.

reconstruction cross-section image. Positively charged residues proximal to the putative phosphotungstate polyanions are labelled. Putative individual tungsten (W) atoms are resolved at most highly occupied binding sites (high densities). The bottom-right extra density is difficult to interpret (question mark) as it occupies an unusual position for phosphotungstate (near M side chain).

a

half maps FSC

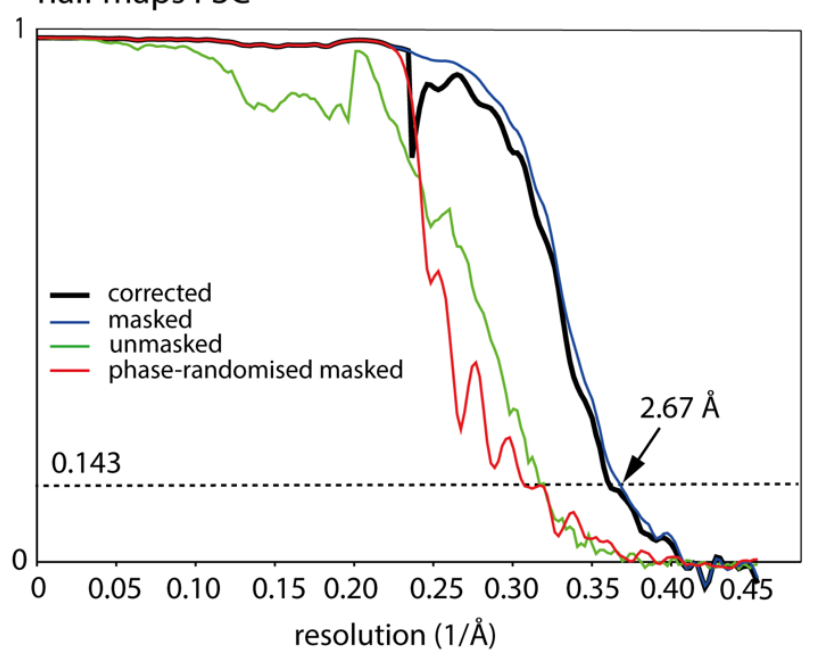

b model:map FSC



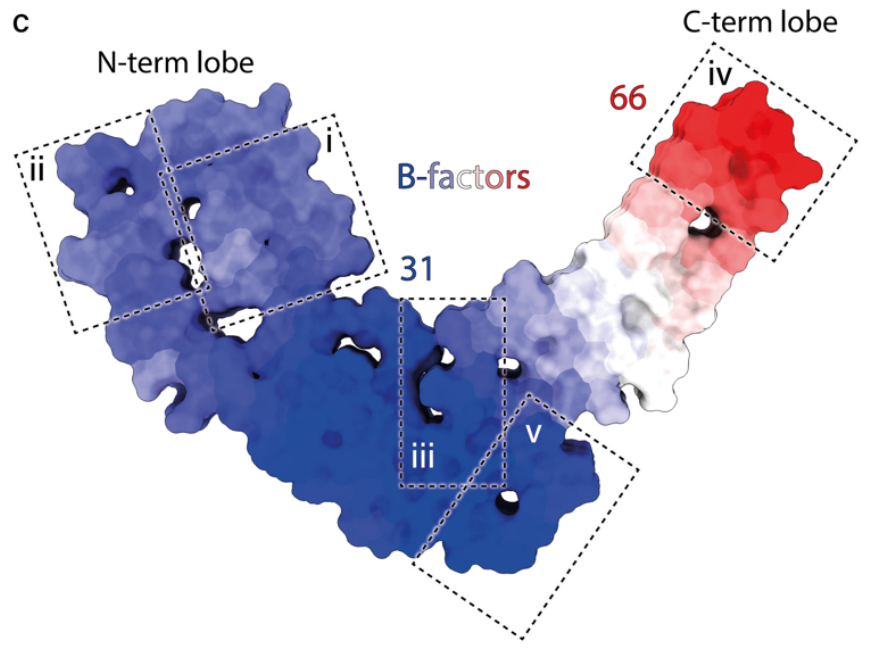

ii

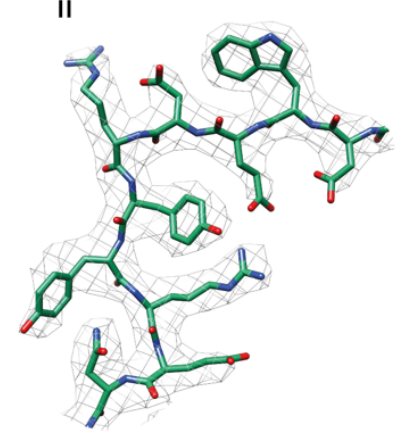

iii

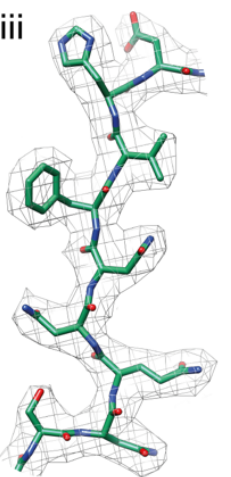

iv

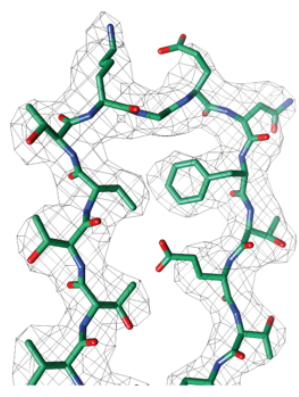

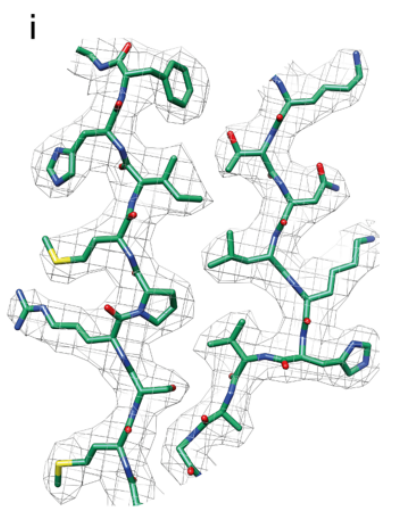

v

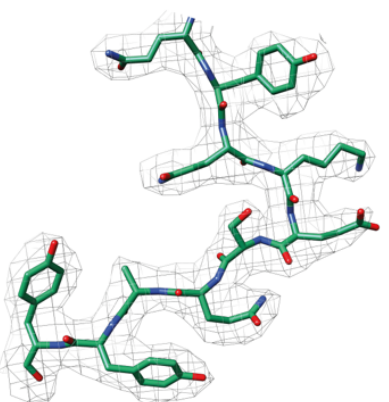

Extended Data Fig. 5. Cryo-EM map resolution and model:map fit. a) Fourier Shell Correlation (FSC) plots as outputted by Relion 3.1. The final plot (black line) is corrected for overfitting with high-resolution noise substitution. b) model:map FSC. c) Atomic B-factor values colour-coded on the solvent-excluded model surface and close-up views (i-v) of model:map fit in selected regions (C, green; N, blue; O, red; S, yellow; map, wireframe). 


\section{References}

1 Prusiner, S. B. Prions. Proc Natl Acad Sci USA 95, 13363-13383 (1998).

2 Collinge, J. \& Clarke, A. A general model of prion strains and their pathogenicity. Science 318, 930-936 (2007).

3 Collinge, J. Mammalian prions and their wider relevance in neurodegenerative diseases. Nature 539, 217-226 (2016).

4 Terry, C. \& Wadsworth, J. D. F. Recent advances in understanding mammalian prion structure: A mini review. Front Mol Neurosci 12, 169 (2019).

5 Prusiner, S. B. Biology and genetics of prions causing neurodegeneration. Annu Rev Genet 47, 601-623 (2013).

6 Goedert, M. NEURODEGENERATION. Alzheimer's and Parkinson's diseases: The prion concept in relation to assembled Abeta, tau, and alpha-synuclein. Science 349, 1255555 (2015).

7 Walker, L. C. Proteopathic strains and the heterogeneity of neurodegenerative diseases. Annu Rev Genet 50, 329-346 (2016).

8 Jaunmuktane, Z. et al. Evidence for human transmission of amyloid-beta pathology and cerebral amyloid angiopathy. Nature 525, 247-250 (2015).

9 Qiang, W., Yau, W. M., Lu, J. X., Collinge, J., \& Tycko, R. Structural variation in amyloidbeta fibrils from Alzheimer's disease clinical subtypes. Nature 541, 217-221 (2017).

10 Condello, C. et al. Structural heterogeneity and intersubject variability of Abeta in familial and sporadic Alzheimer's disease. Proc Natl Acad Sci USA 115, E782-E791 (2018).

11 Peng, C., Gathagan, R. J., \& Lee, V. M. Distinct alpha-synuclein strains and implications for heterogeneity among alpha-synucleinopathies. Neurobiol Dis 109, 209-218 (2018).

12 Purro, S. A. et al. Transmission of amyloid-beta protein pathology from cadaveric pituitary growth hormone. Nature 564, 415-419 (2018).

13 Vaquer-Alicea, J. \& Diamond, M. I. Propagation of protein aggregation in neurodegenerative diseases. Annu Rev Biochem 88, 785-810 (2019).

14 Fitzpatrick, A. W. P. et al. Cryo-EM structures of tau filaments from Alzheimer's disease. Nature 547, 185-190 (2017).

15 Falcon, B. et al. Structures of filaments from Pick's disease reveal a novel tau protein fold. Nature 561, 137-140 (2018).

16 Falcon, B. et al. Novel tau filament fold in chronic traumatic encephalopathy encloses hydrophobic molecules. Nature 568, 420-423 (2019). 
17 Zhang, W. et al. Novel tau filament fold in corticobasal degeneration. Nature 580, 283287 (2020).

$18 \mathrm{Shi}, \mathrm{Y}$. et al. Structure-based classification of tauopathies. Nature 598, 359-363 (2021).

19 Kollmer, M. et al. Cryo-EM structure and polymorphism of Abeta amyloid fibrils purified from Alzheimer's brain tissue. Nat Commun 10, 4760 (2019).

20 Yang, Y. et al. Cryo-EM structures of amyloid- $\beta 42$ filaments from human brain. bioRxiv 2021.10.19.464936; doi: https://doi.org/10.1101/2021.10.19.464936 (2021).

21 Schweighauser, M. et al. Structures of alpha-synuclein filaments from multiple system atrophy. Nature 585, 464-469 (2020).

22 Meyer, R. K. et al. Separation and properties of cellular and scrapie prion proteins. Proc Natl Acad Sci USA. 83, 2310-2314 (1986).

23 Prusiner, S. B. Molecular biology of prion diseases. Science 252, 1515-1522 (1991).

24 Hosszu, L. L. P. et al. Structural mobility of the human prion protein probed by backbone hydrogen exchange. Nature Struct Biol 6, 740-743 (1999).

25 Wuthrich, K. \& Riek, R. Three-dimensional structures of prion proteins. Adv Protein Chem 57, 55-82 (2001).

26 Rodriguez, J. A., Jiang, L., \& Eisenberg, D. S. Toward the atomic structure of PrPSc. Cold Spring Harb Perspect Biol. 9, a031336. doi: 10.1101/cshperspect.a031336. (2017).

27 Pan, K. M. et al. Conversion of $\alpha$-helices into $\beta$-sheets features in the formation of the scrapie prion proteins. Proc Natl Acad Sci USA 90, 10962-10966 (1993).

28 Caughey, B., Raymond, G. J., \& Bessen, R. A. Strain-dependent differences in $\beta$-sheet conformations of abnormal prion protein. J Biol Chem 273, 32230-32235 (1998).

29 Riesner, D. Biochemistry and structure of $\operatorname{PrP}(C)$ and $\operatorname{PrP}(\mathrm{Sc}) . \mathrm{Br}$ Med Bull 66, 21-33 (2003).

30 Prusiner, S. B. et al. Scrapie prions aggregate to form amyloid-like birefringent rods. Cell 35, 349-358 (1983).

31 Baskakov, I. V. et al. The Prion 2018 round tables (I): The structure of $\operatorname{PrP}(\mathrm{Sc})$. Prion 13, 46-52 (2019).

32 Li, J., Browning, S., Mahal, S. P., Oelschlegel, A. M., \& Weissmann, C. Darwinian evolution of prions in cell culture. Science 327, 869-872 (2010).

33 Collinge, J., Sidle, K. C., Meads, J., Ironside, J., \& Hill, A. F. Molecular analysis of prion strain variation and the aetiology of 'new variant' CJD. Nature 383, 685-690 (1996). 
34 Hill, A. F. et al. Molecular classification of sporadic Creutzfeldt-Jakob disease. Brain 126, 1333-1346 (2003).

35 Khalili-Shirazi, A. et al. PrP glycoforms are associated in a strain-specific ratio in native PrPsc. J Gen Virol 86, 2635-2644 (2005).

36 Hill, A. F. et al. Distinct glycoform ratios of protease resistant prion protein associated with PRNP point mutations. Brain 129, 676-685 (2006).

37 Wadsworth, J. D., Asante, E. A., \& Collinge, J. Contribution of transgenic models to understanding human prion disease. Neuropathol Appl Neurobiol 36, 576-597 (2010).

38 Terry, C. et al. Ex vivo mammalian prions are formed of paired double helical prion protein fibrils. Open Biol 6, 160035 (2016).

39 He, S. \& Scheres, S. H. W. Helical reconstruction in RELION. J Struct Biol 198, 163-176 (2017).

40 Zivanov, J. et al. New tools for automated high-resolution cryo-EM structure determination in RELION-3. Elife 7, (2018).

41 Scheres, S. H. W. Amyloid structure determination in RELION-3.1. Acta Crystallogr D Struct Biol 76, 94-101 (2020).

42 Eisenberg, D. S. \& Sawaya, M. R. Structural studies of amyloid proteins at the molecular level. Annu Rev Biochem 86, 69-95 (2017).

43 ladanza, M. G., Jackson, M. P., Hewitt, E. W., Ranson, N. A., \& Radford, S. E. A new era for understanding amyloid structures and disease. Nat Rev Mol Cell Biol 19, 755-773 (2018).

44 Fitzpatrick, A. W. \& Saibil, H. R. Cryo-EM of amyloid fibrils and cellular aggregates. Curr Opin Struct Biol 58, 34-42 (2019).

45 Gallardo, R., Ranson, N. A., \& Radford, S. E. Amyloid structures: much more than just a cross- $\beta$ fold. Curr Opin Struct Biol 60, 7-16 (2020).

46 Groveman, B. R. et al. Parallel in-register intermolecular beta-sheet architectures for prion-seeded prion protein (PrP) amyloids. J Biol Chem 289, 24129-24142 (2014).

47 Wang, L. Q. et al. Cryo-EM structure of an amyloid fibril formed by full-length human prion protein. Nat Struct Mol Biol 27, 598-602 (2020).

48 Glynn, C. et al. Cryo-EM structure of a human prion fibril with a hydrophobic, proteaseresistant core. Nat Struct Mol Biol 27, 417-423 (2020).

49 Kraus, A. et al. High-resolution structure and strain comparison of infectious mammalian prions. Mol Cell 81, 4540-4551 (2021). 
50 Wang, L. Q. et al. Genetic prion disease-related mutation E196K displays a novel amyloid fibril structure revealed by cryo-EM. Sci Adv 7, eabg9676 (2021).

51 Schmidt, C. et al. A systematic investigation of production of synthetic prions from recombinant prion protein. Open Bio/ 5, 150165 (2015).

52 Chandler, R. L. Encephalopathy in mice produced by inoculation with scrapie brain material. Lancet 1, 1378-1379 (1961).

53 Scott, M. et al. Transgenic mice expressing hamster prion protein produce speciesspecific scrapie infectivity and amyloid plaques. Cell 59, 847-857 (1989).

54 Wenborn, A. et al. A novel and rapid method for obtaining high titre intact prion strains from mammalian brain. Sci Rep 5, 10062 (2015).

55 Terry, C. et al. Structural features distinguishing infectious ex vivo mammalian prions from non-infectious fibrillar assemblies generated in vitro. Sci Rep 9, 376 (2019).

56 Klohn, P., Stoltze, L., Flechsig, E., Enari, M., \& Weissmann, C. A quantitative, highly sensitive cell-based infectivity assay for mouse scrapie prions. Proc Natl Acad Sci USA 100, 11666-11671 (2003).

57 Safar, J. et al. Eight prion strains have $\mathrm{PrP}^{\mathrm{Sc}}$ molecules with different conformations. Nat Med 4, 1157-1165 (1998).

58 Levine, D. J. et al. Mechanism of scrapie prion precipitation with phosphotungstate anions. ACS Chem Biol 10, 1269-1277 (2015).

59 Keggin, J. F. The structure and formula of 12-phosphotungstic acid. Proc $R$ Soc Lond $A$ 144, 75-100 (1934).

60 Vazquez-Fernandez, E. et al. The structural architecture of an infectious mammalian prion using electron cryomicroscopy. PLoS Pathog 12, e1005835 (2016).

61 Artikis, E., Roy, A., Verli, H., Cordeiro, Y., \& Caughey, B. Accommodation of in-register Nlinked glycans on prion protein amyloid cores. ACS Chem Neurosci 11, 4092-4097 (2020).

62 Kamali-Jamil, R. et al. The ultrastructure of infectious L-type bovine spongiform encephalopathy prions constrains molecular models. PLoS Pathog 17, e1009628 (2021).

63 Zhang, K. Gctf: Real-time CTF determination and correction. J Struct Biol 193, 1-12 (2016).

64 Wagner, T. et al. SPHIRE-crYOLO is a fast and accurate fully automated particle picker for cryo-EM. Commun Biol 2, 218 (2019).

65 Zivanov, J., Nakane, T., \& Scheres, S. H. W. A Bayesian approach to beam-induced motion correction in cryo-EM single-particle analysis. IUCrJ 6, 5-17 (2019). 
66 Zivanov, J., Nakane, T., \& Scheres, S. H. W. Estimation of high-order aberrations and anisotropic magnification from cryo-EM data sets in RELION-3.1. IUCrJ 7, 253-267 (2020).

67 Pettersen, E. F. et al. UCSF Chimera--a visualization system for exploratory research and analysis. J Comput Chem 25, 1605-1612 (2004).

68 Emsley, P., Lohkamp, B., Scott, W. G., \& Cowtan, K. Features and development of Coot. Acta Crystallogr D Biol Crystallogr 66, 486-501 (2010).

69 Afonine, P. V. et al. Real-space refinement in PHENIX for cryo-EM and crystallography. Acta Crystallogr. D Struct Biol 74, 531-544 (2018).

70 Murshudov, G. N. et al. REFMAC5 for the refinement of macromolecular crystal structures. Acta Crystallogr. D Biol Crystallogr 67, 355-367 (2011).

71 Williams, C. J. et al. MolProbity: More and better reference data for improved all-atom structure validation. Protein Sci 27, 293-315 (2018).

72 Croll, T. I. ISOLDE: a physically realistic environment for model building into lowresolution electron-density maps. Acta Crystallogr D Struct Biol 74, 519-530 (2018).

73 Pettersen, E. F. et al. UCSF ChimeraX: Structure visualization for researchers, educators, and developers. Protein Sci 30, 70-82 (2021).

74 Wagner, T. et al. Two particle-picking procedures for filamentous proteins: SPHIREcrYOLO filament mode and SPHIRE-STRIPER. Acta Crystallogr D Struct Biol 76, 613-620 (2020). 